\title{
Age Related Changes in Common Bile Duct Transverse Diameter Among Egyptian Population: Ultrsonographic and Magnetic Resonance Study
}

\author{
RADWA M. AHMED, M.D. ${ }^{1}$; ESSAM A. HASSAN, M.D. ${ }^{2}$; MOHAMED F. ZAIDAN, M.D. ${ }^{3}$; \\ MAHMOUD ABOELNOR MOHAMED, M.D. ${ }^{4}$; AMAL M. MAHER, M.D. ${ }^{5}$ and \\ EMAN A. ABD EL-MEGUID, M.D. ${ }^{1}$ \\ The Departments of Anatomy \& Embryology ${ }^{1}$, Tropical Medicine ${ }^{2}$, General Surgery ${ }^{3}$, \\ Radiodiagnosis Diagnostic Radiology ${ }^{4}$ and Internal Medicine , Faculty of Medicine, Fayoum University
}

\begin{abstract}
Background: Measurement of transverse diameter of the Common Bile Duct (CBD) was performed in several studies by Ultrasonography (US) and magnetic resonance imaging using Magnetic Resonance Cholangiopancreatography (MRCP). The size of the extrahepatic bile duct increases with age which should be taken into consideration by the surgeons during the surgery of the gallbladder, pancreas and duodenum. US plays a crucial role in the initial imaging work-up of a patient with jaundice. Because a dilated extrahepatic duct distinguishes obstructive from non-obstructive causes of jaundice. The size of the bile duct is considered to increase normally with age as proved by various studies especially MRCP which is more accurate than US.
\end{abstract}

Aim of Study: The aim of the present work is to demonstrate anatomical variation concerning assessment of transverse diameter of CBD both in adult and geriatric age groups without biliary or hepatic manifestations and detection of age related changes in $\mathrm{CBD}$ as one of the causes of non obstructive biliiary dilatation.

Material and Methods: The patients were divided into three groups according to their age: 1- Group I: 21-40 years. 2- Group II: 41-60 years, 3- Group III: Over 60 years. Ultrasound and MRCP techniques were used in this study to assess the diameter of CBD in different age groups.

Results: Group I: In US: In this age group the mean of the widest transverse diameter of extrahepatic bile duct was 0.25 with a range: $0.18-0.41$. In males the mean was: 0.23 with a range: $0.19-0.26$. In females: The mean was: 0.28 with a range: $0.18-0.41$. In MRCP: In this age group the mean of the widest transverse diameter of extrahepatic bile duct was 37.5 with a range: $21-49$. In males: The mean was: 36 with a range: $21-46$. In females: The mean was: 40 with a range: 0.29-49. Group II: In US: The mean of the widest transverse diameter of extrahepatic bile duct was 0.32 with a range: $0.19-0.41$. In males: The mean was: 0.31 with a range: 0.19 0.39 . In females: The mean was: 0.33 with a range: 0.27 -

Correspondence to: Dr. Radwa M. Ahmed, The Department of Anatomy \& Embryology, Faculty of Medicine,

Fayoum University
0.41. In MRCP: The mean of the widest transverse diameter of extrahepatic bile duct was 46.5 with a range: $24-62$. In males: The mean was: 52.6 with a range: $41-62$. In females: The mean was: 40.4 with a range: $0.24-51$. Group III: In US: The mean of the widest transverse diameter of extrahepatic bile duct was 0.40 with a range: $0.26-0.63$. In males: The mean was: 0.39 with a range: $0.28-0.52$. In females: The mean was: 0.42 with a range: $0.26-0.63$. In MRCP: The mean of the widest transverse diameter of extrahepatic bile duct was 88.31 with a range: $49-180$. In males: The mean was: 94.27 with a range: $69-170$. In females: The mean was: 81.93 with a range: 49-180.

Conclusion: Both US and MRCP modalities revealed that the widest transverse diameter of CBD increases with age. Key Words: Common bile duct diameter-Ultrasonography
- MRCP.

\section{Introduction}

CBD consists of four parts: Supra-duodenal, retroduodenal, retro-pancreatic and intra-duodenal. The supra-duodenal segment found in the right border of the lesser omentum and is related posteriorly to the portal vein, via a thin layer of connective tissue. On the left side of the common bile duct arises the proper hepatic artery. The retroduodenal segment is a part of the anterior wall of the Winslow's hiatus. CBD descends posterior to the superior segment of the duodenum, in relation with the superior duodenal flexure, and describes an arch with anterior concavity. This segment has important vascular relations; the portal vein lies posterior to it, the common hepatic artery lies on the left side of CBD and it will continue with the proper hepatic artery, gastroduodenal artery descends anterior to the portal vein and to the left of the common bile duct. The retropancreatic part, CBD descends through a groove or a canal posterior to the pan- 
creas. Intra-duodenal segment CBD passes through medial wall of the second part of the duodenum and opens together with the main pancreatic duct at the level of the hepato-pancreatic ampulla where CBD elevates the mucosa of the duodenum and forms the longitudinal fold of duodenum. In the inferior end of this fold, there is a prominence-the major duodenal papilla where the hepato-pancreatic ampulla is found. CBD opens into middle third of the duodenum [1,2].

Several studies have been performed quantifying the normal transverse diameter of the CBD measured by Ultrasosonography (US) [3] . Transabdominal ultrasound is one of the imaging modalities routinely used in the evaluation of the extra-hepatic biliary system diseases because it is noninvasive and inexpensive imaging method. Ultrasound is still the first imaging study widely used to evaluate the biliary system pathology $[4,5]$

Among non-obstructive causes of CBD dilatation is opioids consumption as they may cause an increase in the basic pressure and in frequency of phasic contractions of the Oddi's sphincter leading to biliary dilatation. In a study performed by [6] the authors showed an association between increased biliary diameter, evaluated on US, and addiction to opioids in asymptomatic patients, with normal levels of serum bilirubin and alkaline phosphatise tests and absence of obstructive factors on US. Choledocholithiasis is a pathologic condition also able to induce isolated bile duct dilatations with non-specific symptoms or biochemical abnormalities. It develops in about $10 \%-20 \%$ of patients with gallbladder stones, may be asymptomatic in half of cases and CBD stones cannot always be identified by traditional non invasive techniques e.g. US [7].

[8] stated that MRCP is accurate non invasive imaging technique which uses T2 sequence magnetic resonance used for evaluation of the anatomy and pathology of pancreatobiliary system.

MRCP can be used to measure the diameter of the CBD [9]. They measured the normal CBD diameter in 187 patients by MRCP and found that the CBD diameter was significantly correlated only with age.

MRCP is the main diagnostic procedure that determines whether endoscopic retrograde cholangiopancretography is needed, especially when US results are equivocal [8]. [10] reported that the diameter of the CBD changes in response to various factors, including age, cholecystectomy, measurement location, respiration, and body mass index.

\section{Material and Methods}

The patient:

Study population and sampling:

The study population for US consisted of one hundred patients who were referred to Radiology Department of Fayoum Public Hospital from Feb. 2018-May 2020 for regular check-ups by transabdominal US. Those who were older than 20 years were included. Another one hundred patients were recruited who underwent an abdominal MRI for the current study. We recorded the age, sex, medical history, list of medications, total serum cholesterol and liver function tests. MR imaging was performed on the patients after an overnight fast of at least 8 $\mathrm{h}$ prior to the radiological examination. A written consent was obtained from each patient before imaging for ethical considerations.

The patients were divided into three groups according to their age:

1- Group I: 21-40 years.

2- Group II: 41-60 years.

3- Group III: over 60 years.

The inclusion criteria: (1) Normal abdomen. (3) Hepatic or splenic hemangiomas; and (4) Renal cysts.

The exclusion criteria: (1) Pre-existing hepatobiliary and pancreatic surgery. (2) Intra-or retroperitoneal tumors, inflammation or hemorrhagic diseases; (3) Biliary tract stones; (4) Cholecystitis; (5) Cirrhosis of the liver; (6) Ascites; (7) Abnormal liver function tests (total bilirubin, aspartate aminotransferasea, alanine aminotransferase); (8) Current use of medication that causes relaxation of smooth muscle (e.g., calcium blockers and papaverine hydrochloride); and (9) Abnormal total serum cholesterol. (10) Opiods addicts. (11) Cardiovascular diseases. (12) Pregnancy.

\section{Methods:}

1- Ultrasound: [5]:

The study design:

In supine position, first a brief evaluation of the liver, pancreas, gallbladder, and bile ducts was done. Then, on sagittal plan, when the CBD was visualized in longitudinal axis, during deep inspiration, in its proximal portion the anteroposterior distance from inner borders was measured. In cases that the CBD was not visualized properly, the rightside of the patient's body was raised or the intercostal space was used for better visualization. Logic S7 Expert, GE Heathcare, Korea machine was used in the current study. 


\section{2- Magnetic Resonance Cholangiopancreatography $(M R C P)$ [8]:}

MR imaging technique:

All the examinations were performed with a 1.5-T MR scanner with $38 \mathrm{mT} / \mathrm{M}$ gradients and a $120 \mathrm{mT} / \mathrm{M}$-per-second slope (Philips, Intera 1.0 T, Netherlands) using a phased-array torso-pelvis coil. The imaging sequences, including twodimensional coronal and axial Single Shot Fast Spin-echo (SSFSE) T2-weighted imaging, axial respiratory gating Fast-Recovery Fast-Spin Echo (FRFSE) T2-weighted imaging with fat suppression, fast-spoiled gradient-echo T1-weighted imaging with fat suppression, axial spoiled dual gradient-echo T1-weighted in-and outof-phase MR imaging, axial slab three-dimensional (3D) spoiled gradient-echo dynamic contrast-enhanced MR imaging with fat suppression, and SSFSE radial series slab MRCP, were performed when all the patients were at the end of expiration and were holding their breath.

End-expiration MRCP was considered conventional MRCP for each patient. The volunteers also underwent MRCP at the end of inspiration. Coronal and axial SSFSE T2-weighted images were obtained during breath-holding with the following parameters: Echo Time $(\mathrm{TE})=90-100 \mathrm{~ms} ; 2 \mathrm{~s}$ between slice acquisitions; section thickness $=5 \mathrm{~mm}$; intersection gap $=0.5 \mathrm{~mm}$; matrix $=384 \times 224$; onehalf signal acquired; and field of view $(\mathrm{FOV})=33$ $\mathrm{cm} X 33 \mathrm{~cm}$. FRFSE T2-weighted images were obtained with the following parameters: Repetition time (TR) ms/TE ms=10000-12000/90-100, with TR determined by the frequency of respiration; section thickness $=5 \mathrm{~mm}$; intersection gap $=0.5 \mathrm{~mm}$; matrix $=256$ X 192; Number of Signals Acquired $(\mathrm{NSA})=3$; and $\mathrm{FOV}=34 \mathrm{~cm} \times 34 \mathrm{~cm}$. The acquisitions were completed in approximately 3-4min. Radial oblique slab SSFSE images were obtained for end-expiration and end-inspiration MRCP with the following parameters: $\mathrm{TE}=1300 \mathrm{~ms} ; 6 \mathrm{~s}$ between image acquisitions; section thickness $=40 \mathrm{~mm}$; matrix $=384$ X 224; one-half signal acquired; and FOV $=30 \mathrm{~cm} X 30 \mathrm{~cm}$. All of the other routine sequences mentioned above were not used in the analysis presented in this article; thus, we have not listed the parameters for those sequences. It took $35 \mathrm{~min}$ to complete the contrast-enhanced MR imaging.

\section{MR image analysis:}

The original MRI data were loaded onto a workstation ((Philips, Intera 1.0 T, Netherlands)) for review. Two observers (with 4 and 6 years of experience interpreting abdominal MR images) retrospectively and individually reviewed the coro- nal and transverse T2-weighted and MRCP images to evaluate the CBD. The widest diameter of the CBD was measured by placing an electronic caliper perpendicular to the long axis at the widest visible portion of the CBD on end expiration MRCP for all the patients. To study the effect of breath on the diameter of the CBD, the volunteers also underwent end-inspiration MRCP. The measurements on end-inspiration MRCP were taken at the same location as those on end-expiration.

\section{Results}

US measured in $\mathrm{cm}$. However, MRCP measured in $\mathrm{mm}$.

\section{1- Group I (21-40 years):}

\section{Results of ultrasound technique:}

In this age group the mean of the widest transverse diameter of extrahepatic bile duct was 0.25 with a range: $0.18-0.41$. In males the mean was: 0.23 with a range: $0.19-0.26$. However, in females: The mean was: 0.28 with a range: $0.18-0.41$ Figs. (13-15).

A sagittal view of an ultrasound image showed the widest transverse diameter of extrahepatic bile duct of a 29 years old male patient measured $0.32 \mathrm{~cm}$ Fig. (1). However, a sagittal view of an ultrasound image revealed extrahepatic bile duct of a 22 years old female patient measured $0.28 \mathrm{~cm}$ Fig. (2).

\section{Results of MRCP technique:}

In this age group the mean of the widest transverse diameter of extrahepatic bile duct was 37.5 with a range: $21-49$. In males: The mean was: 36 with a range: 21-46. However, in females: the mean was: 40 with a range: $0.29-49$ Figs. (16-18).

A coronal T2-weighted MRCP image of a 39 years old male patient showed the widest transverse diameter of extrahepatic bile duct measured $0.49 \mathrm{~cm}$ Fig. (3). However, a coronal T2-weighted MRCP image of a 38 years old female patient showed the widest transverse diameter of extrahepatic bile duct measured $0.54 \mathrm{~cm}$ despite the patient had no symptoms or signs of biliary disease Fig. (4).

\section{2- Group II (41-60 years): \\ Results of ultrasound technique:}

In this age group the mean of the widest transverse diameter of extrahepatic bile duct was 0.32 with a range: $0.19-0.41$. In males: The mean was: 0.31 with a range: $0.19-0.39$. However, in females: The mean was: 0.33 with a range: $0.27-0.41$ Figs. (13-15). 
A sagittal view of an ultrasound image showed the widest transverse diameter of extrahepatic bile duct of a 46 years old male patient measured $0.18 \mathrm{~cm}$ Fig. (5). However, a sagittal view of an ultrasound image revealed the widest transverse diameter of extrahepatic bile duct of a 46 years old female patient measured $0.40 \mathrm{~cm}$ Fig. (6).

\section{Results of MRCP technique:}

In this age group the mean of the widest transverse diameter of extrahepatic bile duct was 46.5 with a range: $24-62$. In males: The mean was: 52.6 with a range: 41-62. However, in females: The mean was: 40.4 with a range: $0.24-51$ Figs. (1618).

A coronal T2-weighted MRCP image of a 58 years old male patient showed the widest transverse diameter of extrahepatic bile duct measured $0.54 \mathrm{~cm}$ Fig. (7). However, a coronal T2-weighted MRCP image of a 56 years old female patient showed the widest transverse diameter of extrahepatic bile duct measured $0.63 \mathrm{~cm}$ despite the patient had no symptoms or signs of biliary disease Fig. (8).

\section{3- Group III (over 60 years):}

\section{Results of ultrasound technique:}

In this age group the mean of the widest transverse diameter of extrahepatic bile duct was 0.40 with a range: $0.26-0.63$. In males: The mean was: 0.39 with a range: $0.28-0.52$. However, in females: The mean was: 0.42 with a range: $0.26-0.63$ Figs. (13-15).

A sagittal view of an ultrasound image showed the widest transverse diameter of extrahepatic bile duct of a 46 years old male patient measured $0.41 \mathrm{~cm}$ Fig. (9). However, a sagittal view of an ultrasound image revealed the widest transverse diameter of extrahepatic bile duct of a 65 years old female patient measured $0.83 \mathrm{~cm}$. The patient had no symptoms or signs of biliary disease Fig. (10).

\section{Results of MRCP technique:}

In this age group the mean of the widest transverse diameter of extrahepatic bile duct was 88.31 with a range: $49-180$. In males: The mean was: 94.27 with a range: 69-170. However, in females: The mean was: 81.93 with a range: $49-180$ Figs. (16-18).

A coronal T2-weighted MRCP image of a 70 years old male patient showed the widest transverse diameter of extrahepatic bile duct measured $1.58 \mathrm{~cm}$. The patient had no symptoms or signs of biliary disease Fig. (11). However, a coronal T2-weighted
MRCP image of a 69 years old female patient showed the widest transverse diameter of extrahepatic bile duct measured $2.44 \mathrm{~cm}$. The patient had no symptoms or signs of biliary disease Fig. (12).

\section{Statistical analysis:}

The collected data were organized, tabulated and statistically analyzed using SPSS software statistical computer package version 22 (SPSS Inc., USA). For quantitative data, the mean and Standard Deviation (SD) were calculated. Independent- $t$ test or One Way ANOVA test was used in comparing between any two groups or three groups, respectively. For interpretation of results of tests of significance, significance was adopted at $p \leq 0.05$.

CBD by U/S was a statistically significantly higher in age group $>60$ years $(0.40 \pm 0.09)$ than in age group 41-60 years $(0.32 \pm 0.07)$ and $21-40$ years $(0.25 \pm 0.07), p<0.0001$, and $p=0.016$, respectively. Conversely, the difference between age group 2140 and 41-60 was not a statistically significant, $p=0.175$. Although females had higher CBD measurements than males within each age group, the difference was not a statistically significant, $p>0.050$ Figs. (13-15).

CBD by MRCP was statistically significantly higher in age group $>60$ years $(88.31 \pm 30.26)$ than in age group 41-60 years $(46.5 \pm 10.87)$ and $21-40$ years (37.5 \pm 9.49$), p<0.0001$. On the other hand, the difference between age group 21-40 and 4160 was not a statistically significant, $p=0.728$. The difference between females and males within each age group was not a statistically significant, $p>0.050$ Figs. (16-18).

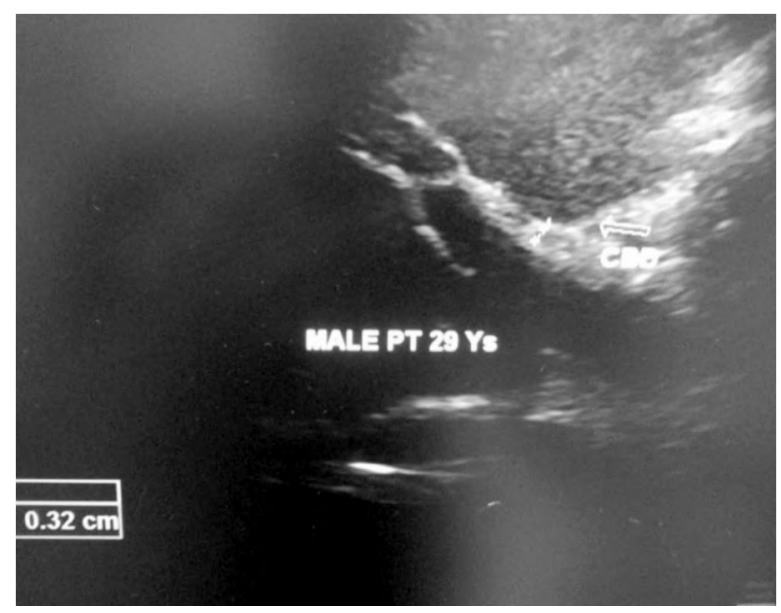

Fig. (1): A sagittal view of an ultrasound image showing the widest transverse diameter of extrahepatic bile duct of a 29 years old male patient measuring $0.32 \mathrm{~cm}$. 


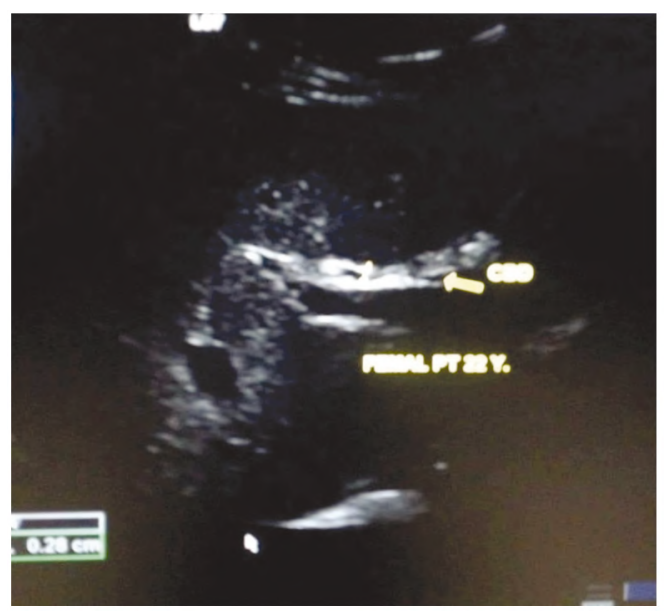

Fig. (2): A sagittal view of an ultrasound image showing the widest transverse diameter of extrahepatic bile duct of a 22 years old female patient measuring $0.28 \mathrm{~cm}$.

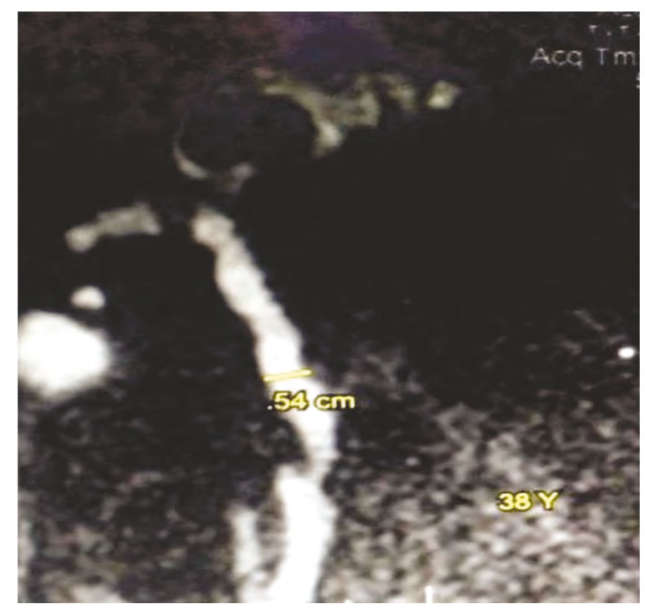

Fig. (4): A coronal T2-weighted MRCP image of a 38 years old female patient showing the widest transverse diameter of extrahepatic common bile duct measuring $0.54 \mathrm{~cm}$. The patient has no symptoms or signs of biliary disease.

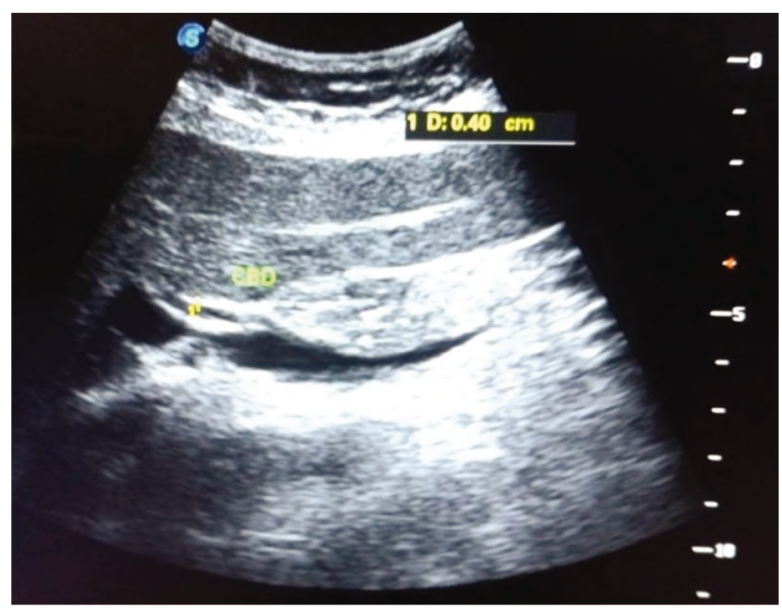

Fig. (6): A sagittal view of an ultrasound image showing the widest transverse diameter of extrahepatic bile duct of a 46 years old female patient measuring $0.40 \mathrm{~cm}$.

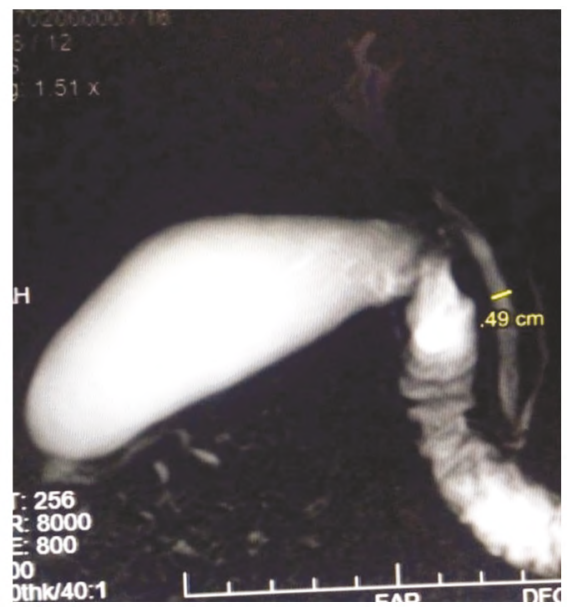

Fig. (3): A coronal T2-weighted MRCP image of a 39 years old male patient showing the widest transverse diameter of extrahepatic common bile duct measuring $0.49 \mathrm{~cm}$.

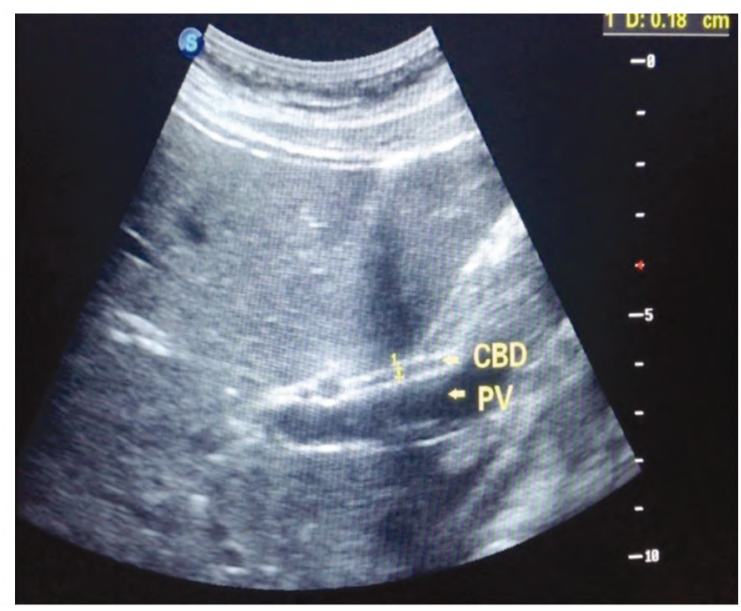

Fig. (5): A sagittal view of an ultrasound image showing the widest transverse diameter of extrahepatic bile duct of a 46 years old male patient measuring $0.18 \mathrm{~cm}$.

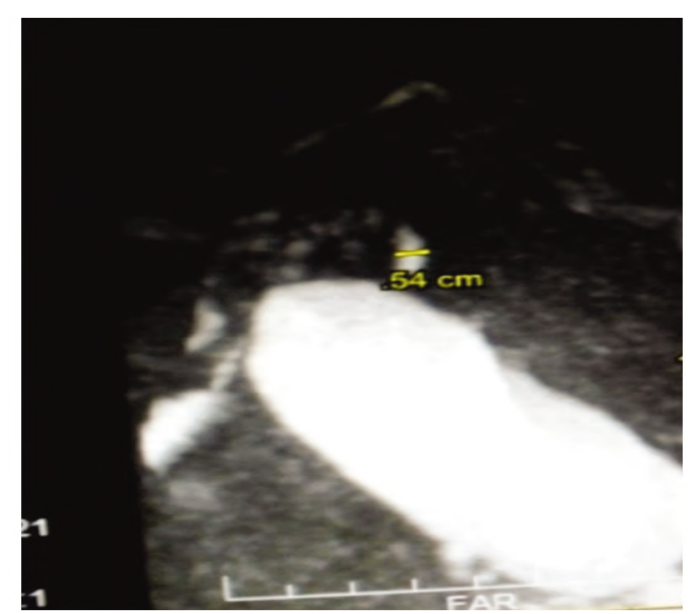

Fig. (7): A coronal T2-weighted MRCP image of a 58 years old male patient showing the widest transverse diameter of extrahepatic bile duct measuring $0.54 \mathrm{~cm}$. 


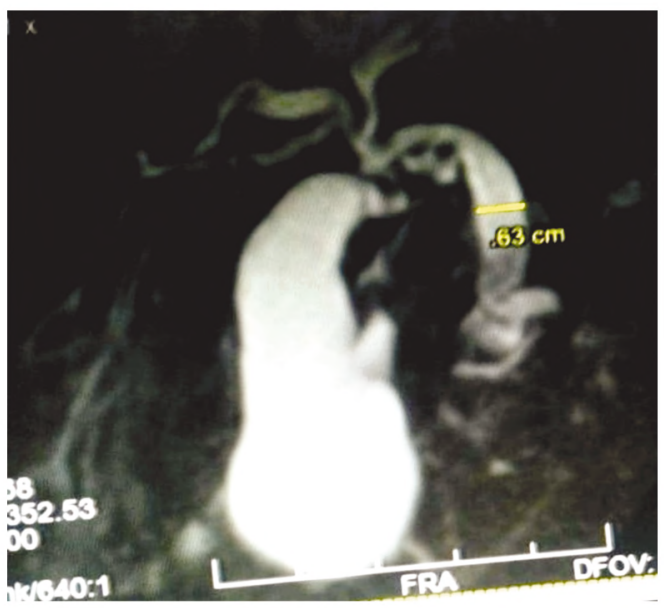

Fig. (8): A coronal T2-weighted MRCP image of a 56 years old female patient showing the widest transverse diameter of extrahepatic bile duct measuring $0.63 \mathrm{~cm}$ The patient has no symptoms or signs of biliary disease.

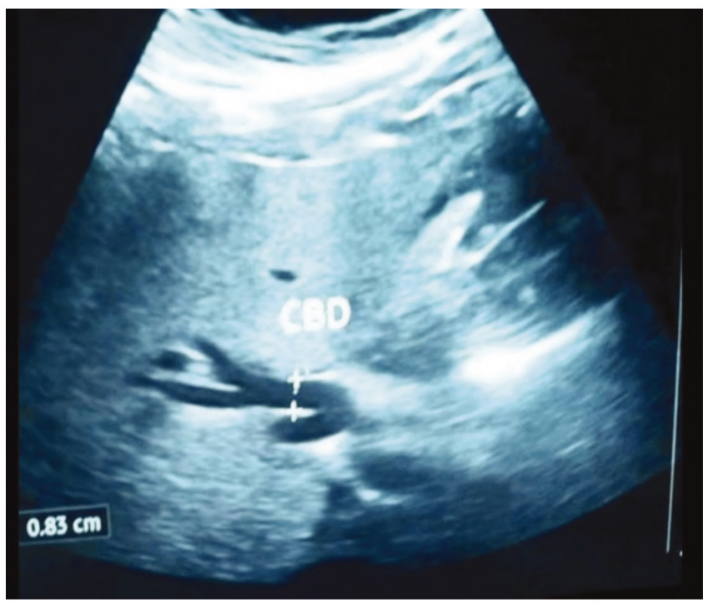

Fig. (10): A sagittal view of an ultrasound image showing the widest transverse diameter of extrahepatic bile duct of a 65 years old female patient measuring $0.83 \mathrm{~cm}$ The patient has no symptoms or signs of biliary disease.

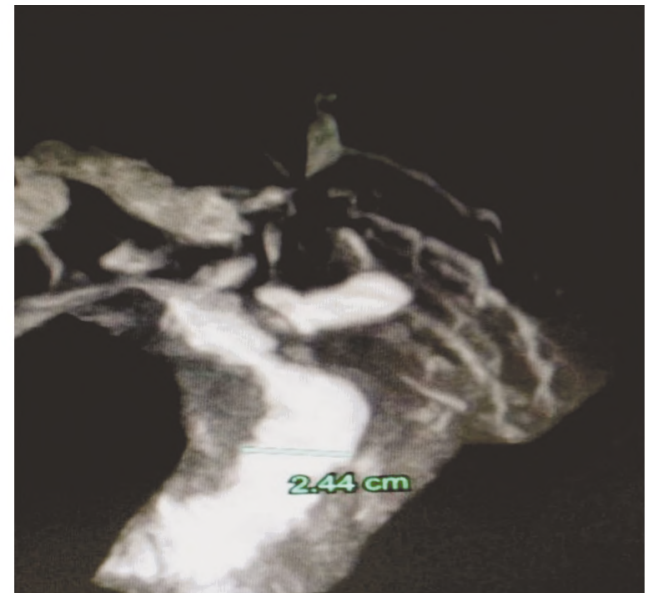

Fig. (12): A coronal T2-weighted MRCP image of a 69 years old female patient showing the widest transverse diameter of extrahepatic bile duct measuring $2.44 \mathrm{~cm}$. The patient has no symptoms or signs of biliary disease.

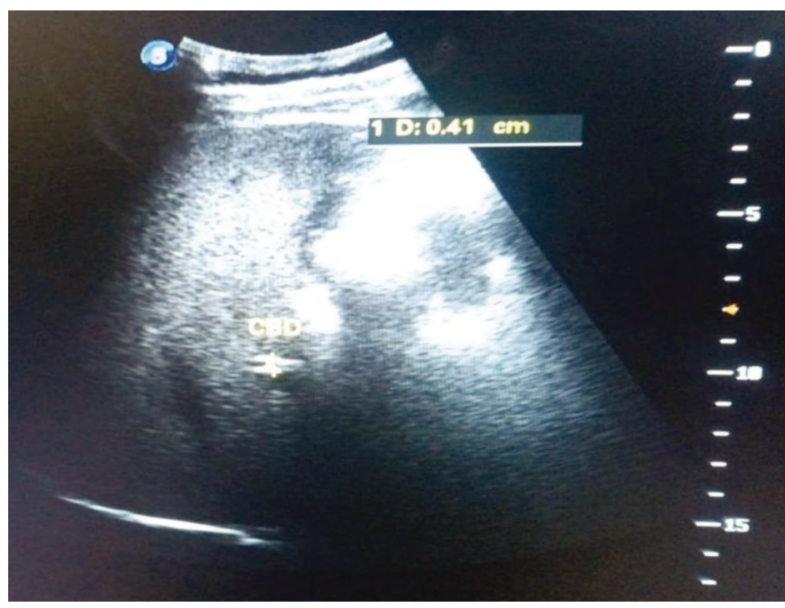

Fig. (9): A sagittal view of an ultrasound image showing the widest transverse diameter of extrahepatic bile duct of a 46 years old male patient measuring $0.41 \mathrm{~cm}$.

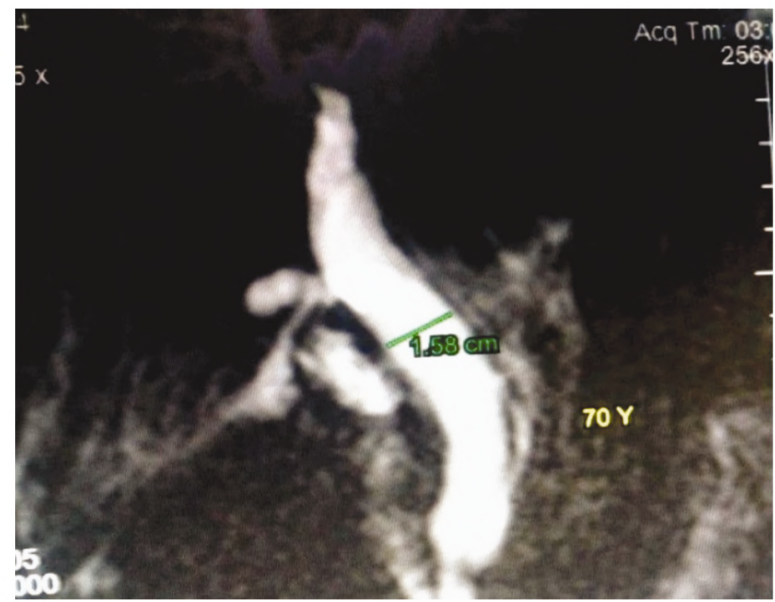

Fig. (11): A coronal T2-weighted MRCP image of a 70 years old male patient showing the widest transverse diameter of extrahepatic bile duct measuring $1.58 \mathrm{~cm}$. The patient has no symptoms or signs of biliary disease.

Fig. (13): A table illustrating mean values of: US measurements of CBD widest transverse diameter among different study groups.

\begin{tabular}{llllll}
\hline & Mean & SD & Minimum Maximum & $p$-value \\
\hline Age: & & & & & \\
$21-40$ & 0.25 & 0.07 & 0.18 & 0.41 & $<0.0001(\mathrm{~S})$ \\
$41-60$ & 0.32 & 0.07 & 0.19 & 0.41 & \\
$>60$ & 0.40 & 0.09 & 0.26 & 0.63 & \\
21-40: & & & & & \\
Male & 0.23 & 0.03 & 0.19 & 0.26 & $0.212(\mathrm{NS})$ \\
Female & 0.28 & 0.08 & 0.18 & 0.41 & \\
41-60: & & & & & \\
Male & 0.31 & 0.08 & 0.19 & 0.39 & $0.538(\mathrm{NS})$ \\
Female & 0.33 & 0.06 & 0.27 & 0.41 & \\
60: & & & & & \\
Male & 0.39 & 0.08 & 0.28 & 0.52 & $0.510(\mathrm{NS})$ \\
Female & 0.42 & 0.1 & 0.26 & 0.63 & \\
\hline
\end{tabular}




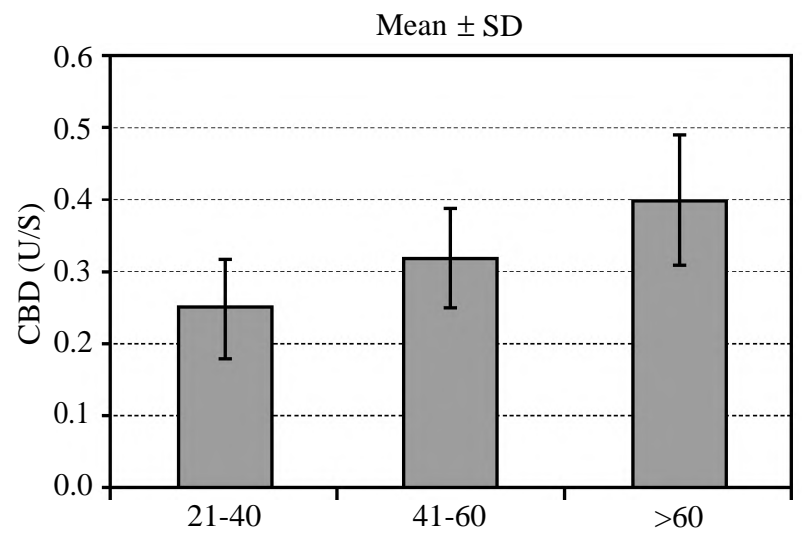

Age groups (years)

Fig. (14): Histogram: Illustrating mean distribution of US measurements of CBD widest transverse diameter among different age groups of the examined patients.

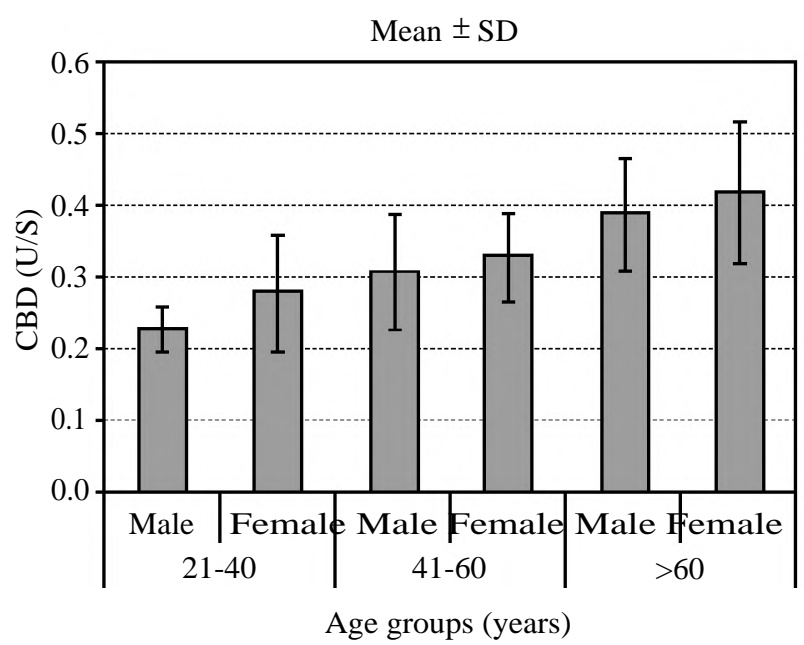

Fig. (15): Histogram: Illustrating mean distribution of US measurements of CBD widest transverse diameter among different age groups in both males and females of the examined patients.

Fig. (16): A table illustrating mean values of: MRCP measurements of CBD widest transverse diameter among different study groups.

\begin{tabular}{|c|c|c|c|c|c|}
\hline \multirow[b]{2}{*}{ Age: } & \multirow[t]{2}{*}{ Mean } & \multirow[t]{2}{*}{ SD } & \multicolumn{2}{|c|}{ Minimum Maximum } & \multirow[t]{2}{*}{$p$-value } \\
\hline & & & & & \\
\hline $21-40$ & 37.5 & 9.49 & 21 & 49 & $<0.0001(\mathrm{~S})$ \\
\hline $41-60$ & 46.5 & 10.87 & 24 & 62 & \\
\hline$>60$ & 88.31 & 30.26 & 49 & 180 & \\
\hline \multicolumn{6}{|l|}{ 21-40: } \\
\hline Male & 36 & 9.92 & 21 & 46 & 0.604 (NS) \\
\hline Female & 40 & 10.15 & 29 & 49 & \\
\hline \multicolumn{6}{|l|}{ 41-60: } \\
\hline Male & 52.6 & 7.5 & 41 & 62 & $0.072(\mathrm{NS})$ \\
\hline Female & 40.4 & 10.78 & 24 & 51 & \\
\hline \multicolumn{6}{|l|}{$>60:$} \\
\hline Male & 94.27 & 26.87 & 69 & 170 & 0.280 (NS) \\
\hline Female & 81.93 & 33.32 & 49 & 180 & \\
\hline
\end{tabular}

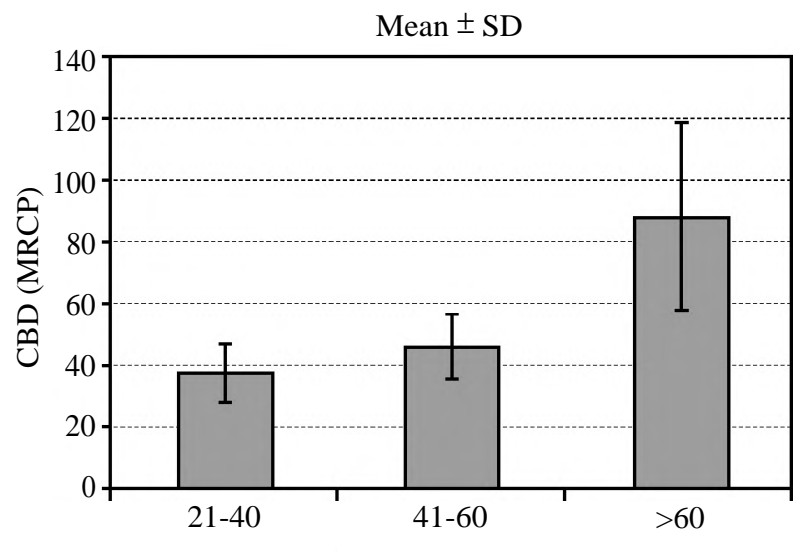

Age groups (years)

Fig. (17): Histogram: Illustrating mean distribution of MRCP measurements of CBD widest transverse diameter among different age groups of the examined patients.

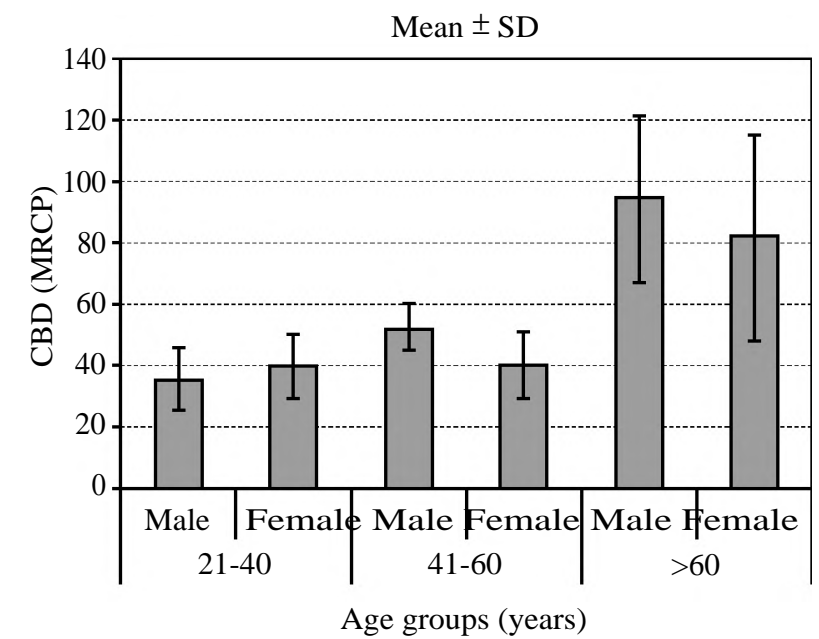

Fig. (18): Histogram: Illustrating mean distribution of MRCP measurements of CBD widest transverse diameter among different age groups in both males and females of the examined patients.

\section{Discussion}

The current study was performed on such a large sample of patients in order to obscure the possible effect of various states of fasting. Although the state of fasting could not be rigidly controlled in this study group, so all patients of the Ultrasound Department were examined at least one hour postprandially.

CBD dilation usually indicates obstructive pathologies such as choleducholithiasis or malignant or benign strictures. The duct dilatation is considered a critical factor in differentiation between obstructive and nonobstructive causes of jaundice. However, non-dilated duct may not necessarily roll out the presence of stones in CBD $[3,11]$. 
Many studies discussed the relationship between aging and CBD diameter variation. The first study was performed in 1984 and demonstrated that that under normal conditions, the inner diameter of the $\mathrm{CBD}$ can be up to $10 \mathrm{~mm}$ and a mild change occurs with aging. Following this report, some researchers found similar results that aging could have significant effect on the CBD size. For instance, a study showed that CBD diameter increased significantly from $3.9 \mathrm{~mm}$ in age range of 18 to 25 years to $4.7 \mathrm{~mm}$ in those older than 55 years 7 . However, other studies did not prove this effect of aging on CBD diameter. However, most of studies concerning this issue revealed that there are variations in the anthropometric characteristics of different races [12-14].

In the current study, US and MRCP studies revealed that the diameters of CBD increase with age both in male and female patients. A similar study performed by [15] concluded that the diameters of CBD increase proportionally with each decade. This enlargement of the CBD diameter in relation to age can be explained by the fragmentation of myocyte bands of the longitudinal smooth muscle, loss of the network of reticular elastic fibers with proximal compensatory dilatation due to distal sclerosis, and interpersed connective tissue. This leads to reduction of contractility and hypotonia of the CBD. Another factor that may be associated is consumption of medications such as calcium channel blockers, nitroglycerin, morphine, and phosphodiesterase type 5 inhibitors which may influence the contractility and tone of the duct. Asympotomatic dilated CBD may lead to decreased or normal levels of alkaline phosphatise enzyme and painless gall stones due to decreased pressure inside as a result of hypotonia of its wall $[7,12,16-$ 19].

Another mechanism was described by [5] who stated that CBD in older persons has a larger size seems to be the result of aging process with less blood flow to the CBD walls which causes abnormalities in tissue metabolism and fragmentation of the smooth muscle which results in decreased contractility of the CBD.

On the other hand, a US study performed by [3] in stated that in majority of patients 60 years old of age or older: The mean diameter of CBD remained being less than $6 \mathrm{~mm}$ in $98 \%$ of patients (1000 of 1018) and less than $7 \mathrm{~mm}$ in $99 \%$ (1015 of 1018) of the patients they surveyed.

In contrast to the current study, a different US study performed by [20] reported that there was no increase in the size of the extrahepatic bile duct with increasing age in an adult population.

\section{Conclusion:}

Both US and MRCP modalities revealed that the transverse diameter of CBD increases with age.

\section{References}

1- STANDRING S.: The Back in Gray's anatomy: The anatomical basis of clinical practices. Gray's Anatomy. 40th ed. New York, USA: Elsevier. Churchill Livingstone, Section 8, Chapter: 69, extrahepatic biliary tree, 2008.

2- BLIDARU D.;, BLIDARU M., POP C., CRIVI C. and SECELEANU A.: The common bile duct: Size, course, relations. Romanian Journal of Morphology and Embryology, 51: 141-4, 2010.

3- PERRET R.S., GREGORY D., SLOOP G.D., JESSICA A. and BORNE J.A.: Common Bile Duct Measurements in an Elderly Population. J. Ultrasound Med., 19: $727-$ 30, 2000.

4- FREITAS M.L., BELL R.L. and DUFFY A.J.: Choledocholithiasis: Evolving standards for diagnosis and management. World J. Gastroenterol., 12 (20): 3162-7, 2006.

5- NALAINI F., SALEHI M.G. and FARSHCHIAN N.: Relationship between age and common bile duct diameter in adults: Ultrasonographic study. International journal of life science and pharma. Research, 4: 20-3, 2017.

6- FARAHMAND H., GHOLAMI P.M. and FATHOLLAH M.S.: Chronic extrahepatic bile duct dilatation: Sonographic screening in the patients with opioid addiction. Korean J. Radiol., 8: 212-5, 2007.

7- ANGELIS C.D., MARIETTI M., BRUNO M., PELLICANO R. and RIZZETTO M.: Endoscopic ultrasound in common bile duct dilatation with normal liver enzymes. World J. Gastrointest. Endosc., 7: 799-805, 2015

8- PENG R., ZHANG L., ZHANG X.M., CHEN T.W., YANG L., HAUNG X.H. and ZHANG Z.M.: Common bile duct diameter in an asymptomatic population: A magnetic resonance imaging study. World Journal of Radiology, 7 (12): 501-8, 2015.

9- CHEN T., HUNG C.R., HUANG A.C., LII J.M. and CHEN R.C.: The diameter of the common bile duct in an asymptomatic Taiwanese population: Measurement by magnetic resonance cholangiopancreatography. J. Chin. Med. Assoc., 75: 384-8, 2012.

10- SENTURK S., MIROGLU T.C., BILICI A., GUMUS H., TEKIN R.C., EKICI F. and TEKBAS G.: Diameters of the common bile duct in adults and postcholecystectomy patients: A study with 64-slice CT. Eur. J. Radiol., 81: 39-42, 2012.

11- MAPLE J.T., BEN-MENACHEM T., ANDERSON M.A., APPALANENI V., BANERJEE S. and CASH B.D.: The role of endoscopy in the evaluation of suspected choledocholithiasis. Gastrointest. Endosc., 71 (1): 1-9, 2010.

12- BACHAR G.N., CHOEN M. and BELENKY A.: Effect of aging on the adult extrahepatic bile duct. A sonographic study. J. Ultrasound Med., 22: 879-82, 2003.

13- LAL N., MEHRA S. and LAL V.: Ultrasonographic measurement of normal common bile duct diameter and 
its correlation with age, sex and anthropometry. J. Clin. Diagn. Res., 8 (12): 1-4, 2014.

14- WORKU M.G., ENYEW E.F., DESITA Z.T. and MOGES A.M.: Sonographic measurement of normal common bile duct diameter and associated factors at the University of Gondar comprehensive specialized hospital and selected private imaging center in Gondar town, North West Ethiopia. Plos One, 15 (1): e0227135, 2020.

15-ZULETA M.A.G., MORALES O.F.R. and REGINO W.O. What is the normal size of the common bile duct. Rev. Colomb. Gastroenterol., 32: 98-104, 2017.

16- SHANMUGAM V., BEATTIE G.C. and YULE S.R.: Is magnetic resonance cholangiopancreatography the new gold standard in biliary imaging? Br. J. Radiol., 78: 88893, 2005.
17- SODICKSON A., MORTELE K.J. and BARISH M.A.: Three-dimensional fast-recovery fast spin-echo MRCP: comparison with two-dimensional single-shot fast spinecho techniques. Radiology, 238: 549-59, 2006.

18- ADMASSIE D.: Ultrasound assessment of common bile duct diameter in Tikur Anbessa Hospital, Addis Ababa, Ethiopia. Ethiop. Med. J., 46: 391-5, 2008.

19- MATCUK G.R., GRANT E.G. and RALLS P.W.: Ultrasound measurements of the bile ducts and gallbladder: Normal ranges and effects of age, sex, cholecystectomy, and pathologic states. Ultrasound, 30: 41-8, 2014.

20- HORROW J.C., NIAKOSARI A., KIRBY C.L. and ROSENBERG H.K.: Gastrointestinal Imaging Aging, Bile ducts anatomy. Radiology, 221: 411-4, 2001.

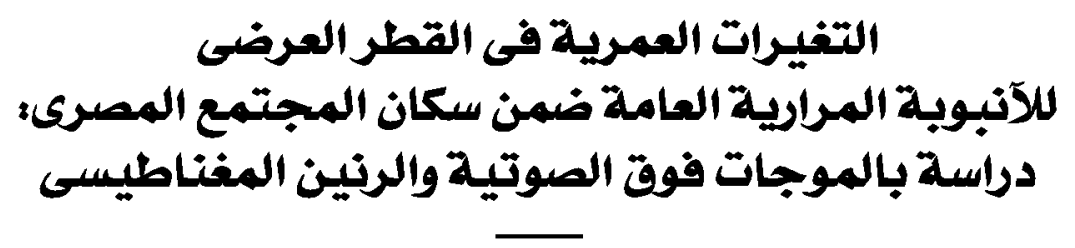

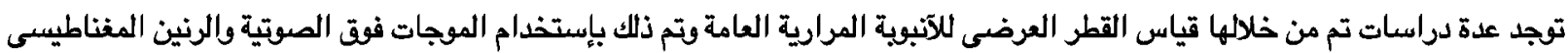

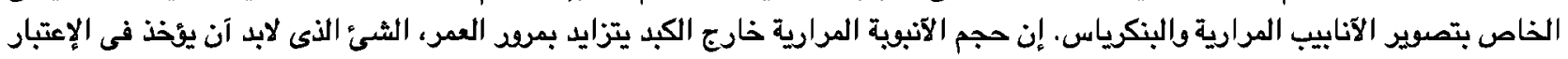

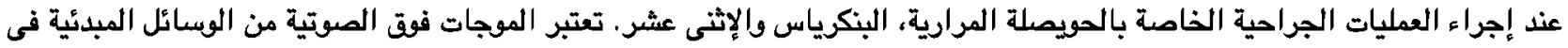

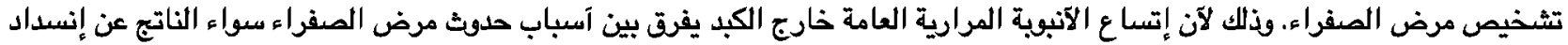

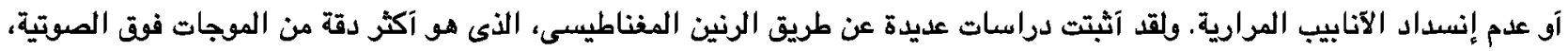

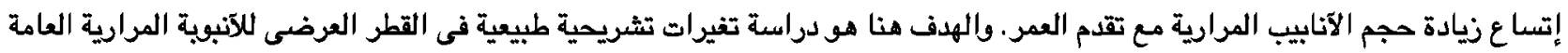

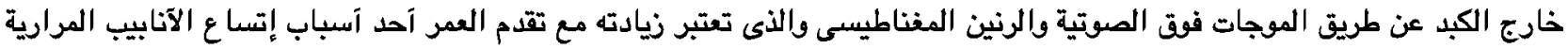

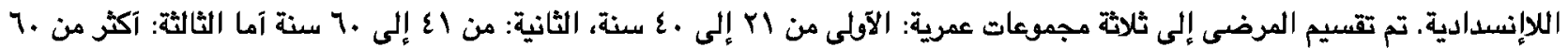

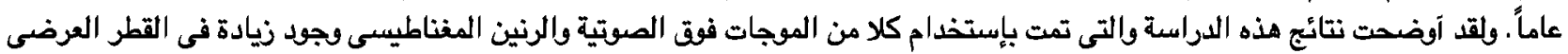

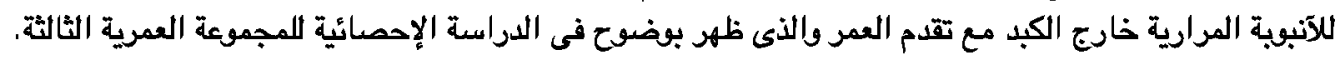

ولقد آثبتت الدراسة الصالية عن طريق الموجات فوق الصوتية والرنين المغناطيسى الخاص بتصوير الآنابيب المرارية والبنكرياس إزدياد

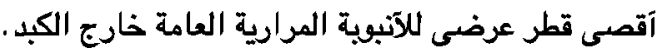

